\title{
Comparative analysis of expressed sequence tags from three castes and two life stages of the termite Reticulitermes flavipes
}

\author{
Matthew M Steller ${ }^{1}$, Srinivas Kambhampati ${ }^{1 *}$, Doina Caragea $^{2}$
}

\begin{abstract}
Background: Termites (Isoptera) are eusocial insects whose colonies consist of morphologically and behaviorally specialized castes of sterile workers and soldiers, and reproductive alates. Previous studies on eusocial insects have indicated that caste differentiation and behavior are underlain by differential gene expression. Although much is known about gene expression in the honey bee, Apis mellifera, termites remain relatively understudied in this regard. Therefore, our objective was to assemble an expressed sequence tag (EST) data base for the eastern subterranean termite, Reticulitermes flavipes, for future gene expression studies.

Results: Soldier, worker, and alate caste and two larval cDNA libraries were constructed, and approximately 15,000 randomly chosen clones were sequenced to compile an EST data base. Putative gene functions were assigned based on a BLASTX Swissprot search. Categorical in silico expression patterns for each library were compared using the R-statistic. A significant proportion of the ESTs of each caste and life stages had no significant similarity to those in existing data bases. All cDNA libraries, including those of non-reproductive worker and soldier castes, contained sequences with putative reproductive functions. Genes that showed a potential expression bias among castes included a putative antibacterial humoral response and translation elongation protein in soldiers and a chemosensory protein in alates.

Conclusions: We have expanded upon the available sequences for $R$. flavipes and utilized an in silico method to compare gene expression in different castes of an eusocial insect. The in silico analysis allowed us to identify several genes which may be differentially expressed and involved in caste differences. These include a gene overrepresented in the alate cDNA library with a predicted function of neurotransmitter secretion or cholesterol absorption and a gene predicted to be involved in protein biosynthesis and ligase activity that was overrepresented in the late larval stage cDNA library. The EST data base and analyses reported here will be a valuable resource for future studies on the genomics of $R$. flavipes and other termites.
\end{abstract}

\section{Background}

Examining the gene expression differences underlying social development and behavior has been termed sociogenomics [1]. Sociogenomics is predicated on two observations: "social life has a biological basis and thereby is influenced by genes and evolution" and "molecular functions of many genes are conserved across species" [1]. Therefore, it is possible to examine eusocial species such as termites, honey bees, ants, and

\footnotetext{
* Correspondence: srini@ksu.edu

'Department of Entomology, Kansas State University, Manhattan, KS 66506, USA

Full list of author information is available at the end of the article
}

social wasps in an ecological and molecular context to better understand the genetics, function, and origins of eusocial behavior.

Termites (Isoptera) are a large and diverse group of eusocial insects. Although eusociality is believed to have evolved only once in Isoptera, the $\sim 2600$ described species show enormous diversity in life history, behavior, colony composition, morphology, physiology, ecology, and biogeography $[2,3]$. Members of the genus Reticulitermes (Rhinotermitidae) are found throughout much of the contiguous United States, with introduced populations in Europe, South America, and the Bahamas [4]. Reticulitermes flavipes Kollar, the eastern subterranean
C Biomed Central

() 2010 Steller et al; licensee BioMed Central Ltd. This is an Open Access article distributed under the terms of the Creative Commons Attribution License (http://creativecommons.org/licenses/by/2.0), which permits unrestricted use, distribution, and reproduction in any medium, provided the original work is properly cited. 
termite, is the most common species in the United States. $R$. flavipes colonies, like those of other termites, consist of morphologically and behaviorally specialized castes. All individuals emerge from eggs as larvae, beyond which the development is flexible in that larvae may develop into nymphs and subsequently into dispersing alates, or into the sterile workers or soldiers [reviewed in ref. [2]]. Workers are involved in nest building, tunnel maintenance, and brood care whereas soldiers defend the colony and possibly communicate general colony fitness. Workers and nymphs of $R$. flavipes may develop into supplementary reproductives under certain conditions [2,3].

Historically, Reticulitermes spp. have been difficult to study mainly due to their cryptic living conditions and amorphous nest structure [2,5]. However, the advent of genomic and bioinformatic methods has provided new opportunities to study these organisms [2]. A macroarray study of $R$. flavipes [6] demonstrated differences in gene expression among the different castes. Genes that exhibited differential expression between workers and soldiers included salivary cellulases, endoglucanases, tubulins, and troponins [6].

Other examples of differential gene expression among termite castes include higher expression of the mitochondrial cytochrome oxidase-III subunit in workers and nymphs of $R$. santonesis [7], increased cytochrome P450 levels after methoprene application in soldier and presoldier fat body in Hodotermopsis sjostedti and Nasutitermes takasagoensis [8,9], and a gene specifically expressed in $H$. japonica soldier mandibles termed SOL1 thought to be involved in soldier specific behavior [10].

In the well-studied eusocial honey bee, Apis mellifera, there is a tendency for behaviorally associated genes to exhibit a $\mathrm{G}+\mathrm{C}$ bias within promoter regions when compared to Drosophila melanogaster and more cis-regulatory elements [11] as well as high levels of expression of ribosomal and hexameric storage proteins in queen and worker cDNA libraries [12]. An EST analysis revealed that honey bee workers showed an over-expression of genes involved in cell differentiation and hydrolase activity, whereas honey bee queens exhibited an upregulation of genes involved in metabolism and oxidoreductase activity [13].

Given that there has been no large-scale effort to identify expressed genes in termites, our first objective was to characterize $R$. flavipes genes by analyzing an EST database of $\sim 15,000$ clones from three cDNA libraries constructed from workers, soldiers, and alates as well as two cDNA libraries constructed from early and late termite larval stages. Our second objective was to undertake an in silico analysis of expression patterns among the castes and life stages to tentatively identify putatively differentially expressed genes.

\section{Methods}

\section{Termites}

Five cDNA libraries were constructed from $R$. flavipes workers, soldiers, alates, and larval stages collected in March 2006 from a single colony on the campus of the University of Florida, Gainesville. The larvae were divided, based on size, into early (putatively stages 1-2) and late (putatively stages 3-4) stages. Several hundred individuals of each caste/stage totaling about $2 \mathrm{gm}$ of wet weight were obtained and stored at $-80^{\circ} \mathrm{C}$ until used. The individuals were not separated according to sex; therefore, the cDNA libraries contained expressed genes in both males and females, although we do not know the precise sex ratio among individuals that were used for mRNA isolation and cDNA synthesis. The larvae have a dichotomous developmental pathway, developing into either nymphs (which then become alates or second-form reproductives) or workers (some proportion of which subsequently become presoldiers and then soldiers).

\section{cDNA Libraries}

mRNA isolation, cDNA synthesis and library construction was undertaken by AGCT Corp. (Cranston, RI). The cDNA was non-normalized and cloned into pBluescript II SK+ vector after digestion with EcoRV and Notl. We did not attempt to systematically exclude bacterial, protozoan, or other symbiont DNA from the libraries. Libraries were plated on LB medium with $50 \mathrm{mg} /$ litre ampicillin and grown at $37^{\circ} \mathrm{C}$ overnight. Several hundred colonies were sampled randomly from the plates for further processing and sequencing. Colonies were placed in $150 \mu \mathrm{l}$ liquid LB medium containing ampicillin, grown overnight at $37^{\circ} \mathrm{C}$, and stored at $-80^{\circ} \mathrm{C}$ after adding $20 \mu \mathrm{l}$ of sterile glycerol. The colonies were then shipped on dry ice to the University of California-Riverside for sequencing the 5 -ends of the clones using the T3 primer.

\section{Sequence Analyses}

Using Sequencher (v4.7, Gene Codes Corp, Ann Arbor, $\mathrm{MI}$ ), we removed the vector sequence and trimmed ends using program defaults. Sequences shorter than 150 bp were discarded from further analysis after trimming. ESTs were organized into contiguous sequences (contigs) using the default parameters in EGassembler [14]. Highly repetitive sequences were masked using the Drosophila RepBase library in EGassembler.

Gene discovery rate for each library was estimated by dividing the number of contiguous sequences by the total number of singleton sequences. Minimum average sequence insert sizes of cDNA clones were estimated by dividing the total number of base pairs sequenced by the number of ESTs in each library. The maximum 
length is limited by the length of sequence; thus, the actual insert size was likely underestimated. Both singletons and contigs were assigned putative functions using a batch BLASTX reference search from the non-redundant protein database using BlastStation (v2.61, TMSoftware, Arcadia, CA), with an e-value $\leq 1 \mathrm{e}^{-10}$. Genes assigned putative housekeeping roles were identified using a list derived from Eisenberg et al. [15]. Gene ontology (GO) enrichment analysis was performed using Blast2GO tool [16]. The functional annotation in Blast2GO was performed in three steps. First, gene sequences were queried against gene or protein databases and potential homologs identified. During the mapping step, known GO annotations of the homologs were retrieved. Finally, the homolog annotations were used to annotate the uncharacterized genes.

For a set of annotated genes (including genes for which the annotations are obtained outside Blast2GO), several tools for statistical and visual analysis are available. Among these, Blast2GO includes a tool for performing enrichment analysis, i.e., the identification of $\mathrm{GO}$ annotations whose abundance is significantly different between two sets of annotated genes. The GO term enrichment analysis functionality is achieved by integrating Blast2GO with Gossip, a software package that employs Fisher's exact test to estimate the significance of associations between two categorical variables, while correcting for multiple testing using FDR (false discovery rate), FWER (family-wise error rate) and single test $p$-value. A set of GO terms that are under- or overrepresented at a specified significance value is obtained as a result of performing the enrichment analysis.

All EST sequences are deposited in dbEST http://ncbi. nlm.nih.gov/dbEST/ under dbEST-Id 65538065-65551007, and in GenBank under accession numbers G0898823G0911765.

\section{In silico transcript abundance}

Relative transcript abundance in the ESTs derived from each of the five cDNA libraries was analyzed using the R-statistic [17]. The R-statistic can be seen as entropy of partitioning contigs (corresponding to genes) among multiple cDNA libraries, and can be used to identify differentially expressed genes as those genes with high $\mathrm{R}$-value [17]. More precisely, the test statistic $\mathrm{R}$ compares the null hypothesis, $\mathrm{L}_{0}$ ("the abundance of a transcript is the same in all libraries"), with the alternative hypothesis, $\mathrm{L}_{1}$ ("the abundance of the transcript in each library is different", i.e., the corresponding gene is differentially expressed), using the log ratio of the two likelihoods, i.e., $\log \left(L_{1} / L_{0}\right)$. Thus, for a particular transcript (contig) $j$, we have:

$$
R_{j}=\sum_{i=1}^{m} x_{i, j} \log \left(\frac{x_{i, j}}{N_{i} f_{j}}\right) f_{j}=\frac{\sum_{i=1}^{m} x_{i, j}}{\sum_{i=1}^{m} N_{i}}
$$

where $m$ is the number of EST libraries considered, $x_{i, j}$ is the number of ESTs in contig $j$ belonging to the $i$ th library, $N_{i}$ is the total number of ESTs in the $i$ th library and $f_{j}$ is the frequency of all of the ESTs of contig $j$ in all of the libraries. If $x_{i, j}=0$ for a library $i$, then the contribution of the $i$ th library to $R_{j}$ is 0 . Compared to other similar methods (e.g., Fisher's exact test), which can only work for two libraries at a time, the R-statistic can work with a multiple libraries. This feature makes the use of the R-statistic desirable for our analysis.

We analyzed the 20 contigs with the highest R-value, including 10 contigs that returned a BLASTX hit with an e-value $\leq 1 \mathrm{e}^{-10}$ and 10 that had no BLAST hit or a hit with an e-value $\geq 1 \mathrm{e}^{-10}$. The cut-off for the number of contigs (genes) differentially expressed was identified by investigating the frequency of R-statistic among the contigs as described [17]. Putative protein functional analysis of the latter group (i.e., those contigs with no BLAST hit or e-value $\geq 1 \mathrm{e}^{-10}$ ) was performed using the protein function predictor [18] which pools sequences with similar structural motifs and assigns GO terms, to obtain an initial indication of gene function. The protein function predictor attempts to predict protein function to a large number of genes than a conventional BLAST search by providing low-resolution function without losing accuracy. We further validated the R-statistic measure of significant difference using a chi-squared analysis.

\section{Results}

\section{General Library Analysis}

We sequenced 4031 worker, 3836 alate, 3548 soldier, 2111 early larval stage, and 1733 late larval stage cDNA clones for a total of 15,259 clones. Each cDNA library had a minimum average insert size ranging from 570 to $1086 \mathrm{bp}$; the gene discovery rate ranged from $47 \%$ to $60 \%$ (Table 1). Each cDNA library had a $70-90 \%$ of genes with no related sequences in public data bases; of the remaining genes, the worker and late larval libraries had the highest proportion of ribosomal sequences and both larval libraries exhibited the highest level of sequence redundancy after BLASTX analysis (Table 1). Each library contained between $5 \%$ and $16 \%$ of sequences that had an e-value of $\leq 1 \mathrm{e}^{-10}$ and were used in the GO analysis. 
Table 1 Basic characteristics of the five cDNA libraries used in this study

\begin{tabular}{lccccc}
\hline & Worker & Alate & Soldier & $\begin{array}{c}\text { Early } \\
\text { Larval }\end{array}$ & $\begin{array}{c}\text { Late } \\
\text { Larval }\end{array}$ \\
\hline $\begin{array}{l}\text { No. clones } \\
\text { sequenced }\end{array}$ & 4031 & 3836 & 3548 & 2111 & 1733 \\
\hline $\begin{array}{l}\text { Total <150 bp } \\
\text { (discarded) }\end{array}$ & 1069 & 330 & 705 & 99 & 113 \\
\hline $\begin{array}{l}\text { Min. mean insert size } \\
\text { (bp) }\end{array}$ & 570 & 1086 & 638 & 681 & 416 \\
\hline $\begin{array}{l}\text { Gene discovery rate } \\
\mathbf{2}\end{array}$ & $60 \%$ & $47 \%$ & $58 \%$ & $56 \%$ & $51 \%$ \\
\hline Unigenes & 1787 & 1639 & 1637 & 1136 & 792 \\
\hline
\end{tabular}

${ }^{1}$ Minimum mean insert size was calculated as the total number of basepairs (after sequence trimming, vector removal, and discarding of sequences less than $150 \mathrm{bp}$ ) divided by the number of clones sequenced.

${ }^{2}$ Gene discovery rate was calculated as the number of contigs divided by total number of clones sequenced.

\section{Sequence Analysis}

Analysis of the ESTs using level two cellular component GO putative terms showed similar proportions of the terms across castes with cell part, cell, and organelle, and organelle part being the most frequent terms assigned to the sequences (Figures 1, 2 and 3). The types and proportions of genes associated with the cellular component terms (Figure 1) were highly similar among the five cDNA libraries with minor differences in proportion. One difference was the absence of genes associated with extracellular part in alate and worker libraries. The GO analysis of level two molecular function terms showed that genes putatively involved in binding and catalytic activity represented the largest $(>60 \%)$ proportion of ESTs in each library (Figure 2). In general, a greater variety of genes was detected in the solider, alate, worker, and early larval libraries relative to the late larval library. The third GO group we investigated was that of biological processes. Genes associated with ten different terms were detected in each of the five cDNA libraries in approximately equal proportions; those associated with cellular and metabolic processes were the most abundant in all five libraries (Figure 3).

Several genes putatively associated with reproductive function were expressed in both sterile and non-sterile castes (Figure 4). Not surprisingly, the alates expressed the highest number and variety of transcripts (seven) associated with reproduction; however, the soldiers (five) and workers (four) also expressed a number of transcripts putatively associated with reproductive functions. Only two transcripts putatively associated with reproductive function were detected in the early larval stage cDNA library and none in the late larval stage cDNA library; the latter finding is not surprising considering we sequenced the fewest number of clones from the late larval stage cDNA library.

Due to the small number of BLASTX hits, we were unable to obtain many GO terms in proportion to the number of total ESTs to use in the statistical analysis of GO terms. Therefore, we chose a less stringent significance threshold than normally utilized with $p$-values $\leq 0.15$; no significant differences in the proportion of $\mathrm{GO}$ terms were detected among the five cDNA libraries. Any differences described above must be interpreted with caution because of the random nature of the clone selection and the differences in the number of clones sequenced from each of the five cDNA libraries.

\section{In Silico Analysis of Genes With Significant BLAST Hits}

As mentioned, the contigs with significant R-statistic values were separated into two groups: those that returned a significant BLASTX hit and those that did not. For the first group (Table 2) the soldier library showed a bias of transcripts that putatively matched a protein from the cricket paralysis virus. Two separate contigs with an abundance in soldier and alate cDNA libraries showed different levels of sequence identity to the same ejaculatory bulb-specific protein in Drosophila melanogaster [Flybase:CG11390] (Figures 5A and 5B). Three other contigs showed a bias within the alate library that matched closely with cytochrome c oxidase (Blattella germanica), cellulase b (Cellulomonas fimi), and ATP synthase subunit a (Drosophila simulans). A number of contigs also showed overrepresentation in the early and late larval stage libraries including cytochrome b (Drosophila sechellia), tropomyosin (Dermatophagoides farinae), troponin c (Tachypleus tridentatus), cytochrome oxidase subunit 3 (Drosophila melanogaster), and $60 \mathrm{~S}$ ribosomal protein 131 (Spodoptera frugiperda).

\section{In Silico Analysis Using Protein Prediction}

The second in silico analysis of the EST libraries (Table 3) utilized the protein function predictor [18]. Proteins associated with anti-bacterial humoral response and translational elongation were two protein functions predicted for contigs with a transcript bias in the soldier cDNA library (Table 3). Putative functions of neurotransmitter secretion or cholesterol absorption (Figure 5C) as well as cell adhesion, and hydrolase activity (not shown) were overrepresented in the alate cDNA library. Predicted protein functions of protein synthesis and ligase activity (Figure 5D) as well as regulation of transcription, metabolism, stress response, cell adhesion, and spermatogenesis (not shown) exhibited a transcript bias in the late larval stage cDNA library.

\section{Discussion}

\section{General Library Analysis}

The gene discovery rate of sequences for each library ranged from $47 \%$ to $60 \%$. The high proportion of genes with no known function in this analysis is likely due to 


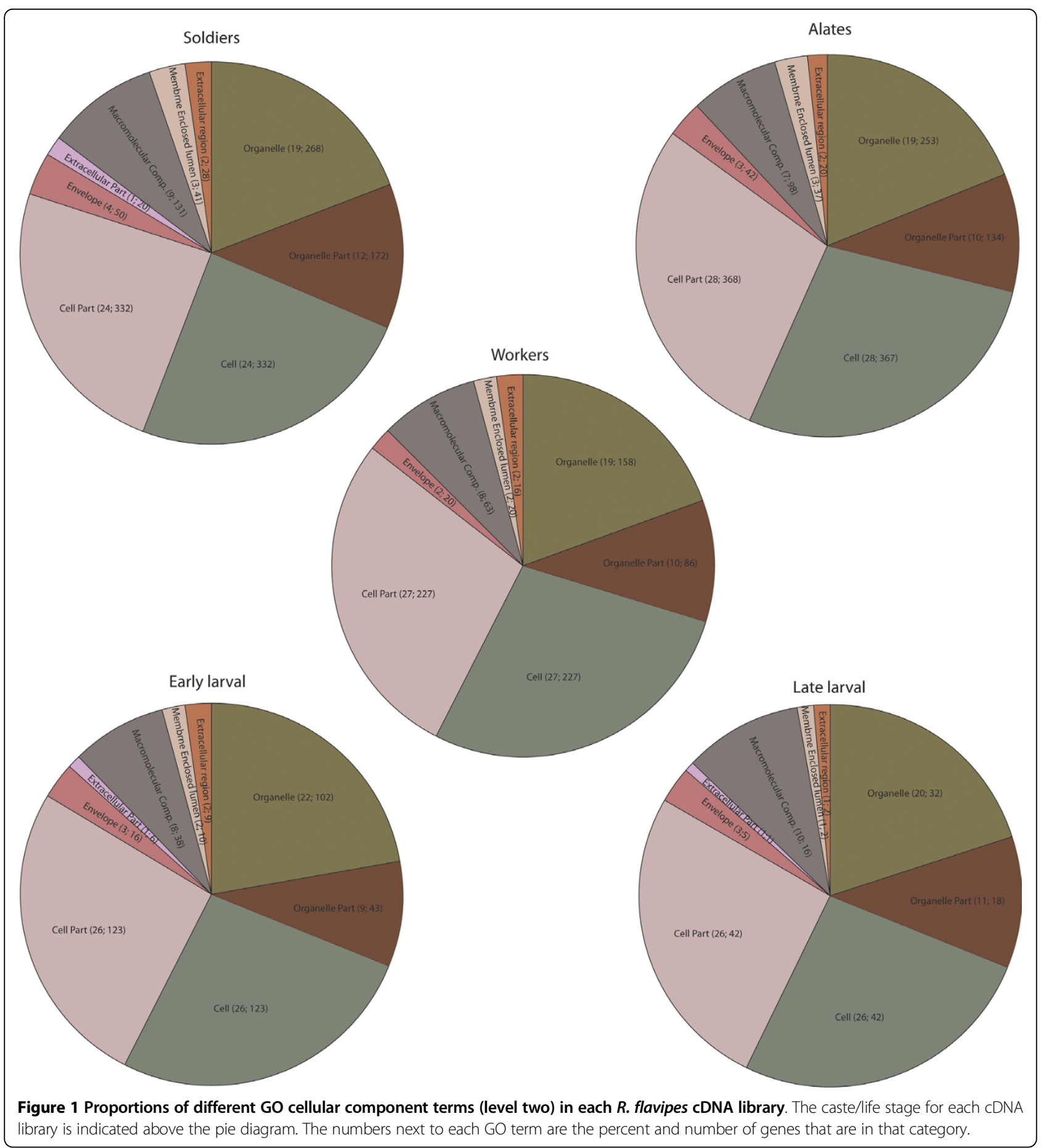

the paucity of termite genes in public databases. In addition, it is possible that some of the unidentified genes may be of protozoan origin because we did not explicitly attempt to exclude protozoa as we prepared the mRNA for cDNA library construction. Finally, some of the sequenced fragments may have been 5' or 3' UTRs or other non-coding regions although a BLAST search for UTRs did not result in an unusually large number of significant hits. In this study, about $12 \%$ of the sequences in the worker and late larval libraries were associated with ribosomal function. A previous study [19] on R. flavipes immatures and adult reproductive castes also found rRNA-like gene expression in workers.

The GO analysis indicated that the proportion and variety of expressed genes in each of the three castes were similar to one another and there were minor 


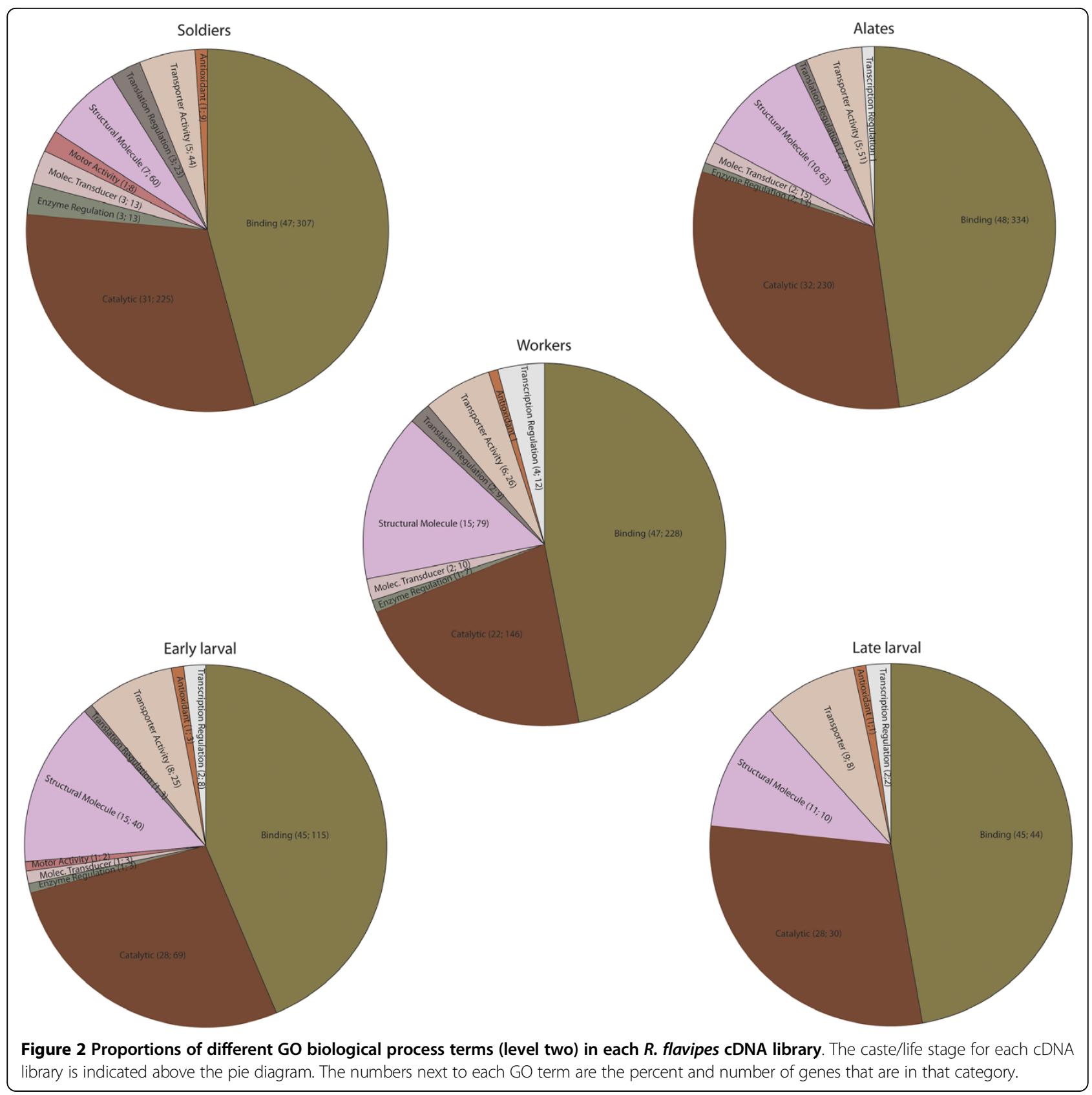

differences among the three castes and the two larval stages. In the analysis involving molecular function terms, the late larval cDNA library exhibited the fewest number of classes of genes. Although this could be a true reflection of differential gene expression patterns, it is possible that the pattern could have resulted due to sequencing the fewest number of clones from the late larval cDNA library relative to the other four libraries. The proportion and variety of genes in the analysis involving biological processes was largely similar among the five cDNA libraries. The fact that most of the GO term representation patterns were similar among the five cDNA libraries suggests that caste-specific differences may involve both transcriptional and posttranscriptional regulation of genes.

In four of the five cDNA libraries (with the exception of late larval library), several genes putatively associated with reproductive functions were expressed. On the surface, the observation that genes associated with reproduction are expressed in individuals of sterile castes such as workers and soldiers may seem counterintuitive. However, the expression of genes involved in reproductive functions in sterile castes is likely a reflection of the fact that $R$. flavipes workers have the ability to become 


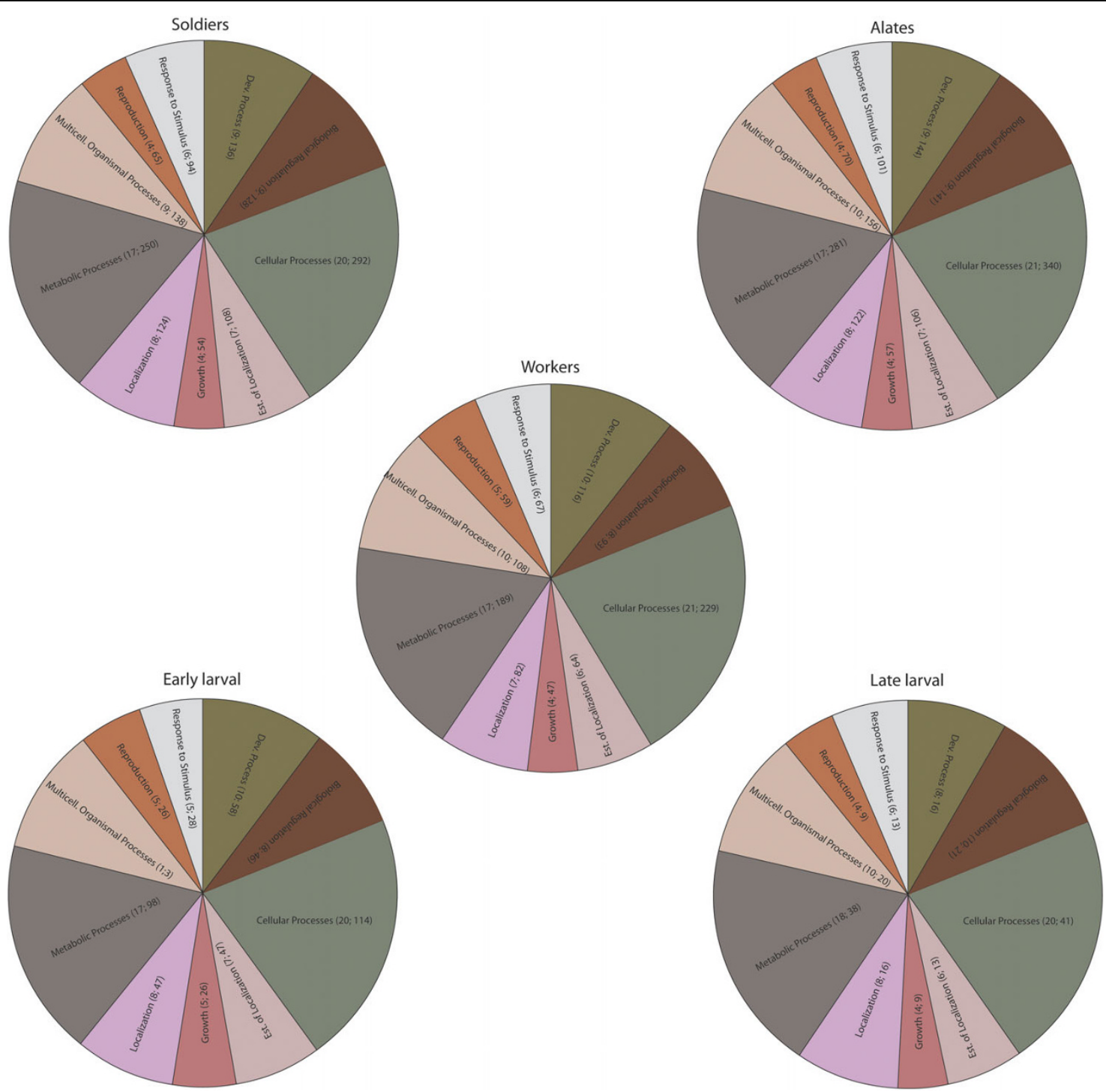

Figure 3 Proportions of different GO molecular function terms (level two) in each $\boldsymbol{R}$. flavipes cDNA library. The caste/life stage for each cDNA library is indicated above the pie diagram. The numbers next to each GO term are the percent and number of genes that are in that category.

third-form reproductives in case of the death of the primary or second-form reproductives [2]. The fact that some of reproductive function genes are also expressed in soldiers, which do not become third-form reproductives, suggests that the genes implicated in reproduction may have other functions unrelated reproduction. Some of the genes involved in reproductive function may be subject to post-transcriptional or post-translational modifications that may render their phenotypic effects undetectable in certain castes or life stages.

\section{In Silico Analysis}

The most bias from the first grouping of sequences used for the in silico analysis was composed mostly of soldier singletons which matched a viral protein, the source of which is not clear. It is possible that the soldiers were infected with a virus or there was viral contamination in the soldier library; alternatively, soldiers could have a protein similar to the viral protein that is expressed at a higher level than in the other castes. This issue needs to be further explored using quantitative real time PCR of individuals from multiple colonies.

Two transcripts that were over-represented in soldier and alate cDNA libraries matched a protein that was similar to the pebIII ejaculatory bulb-specific protein in D. melanogaster. Previous studies suggest that this protein is expressed in pheromone glands of male flies and is similar to an odorant-binding protein in Mamestra brassica that has been implicated in sexual attraction and repulsion [20]; however, recent studies have also 


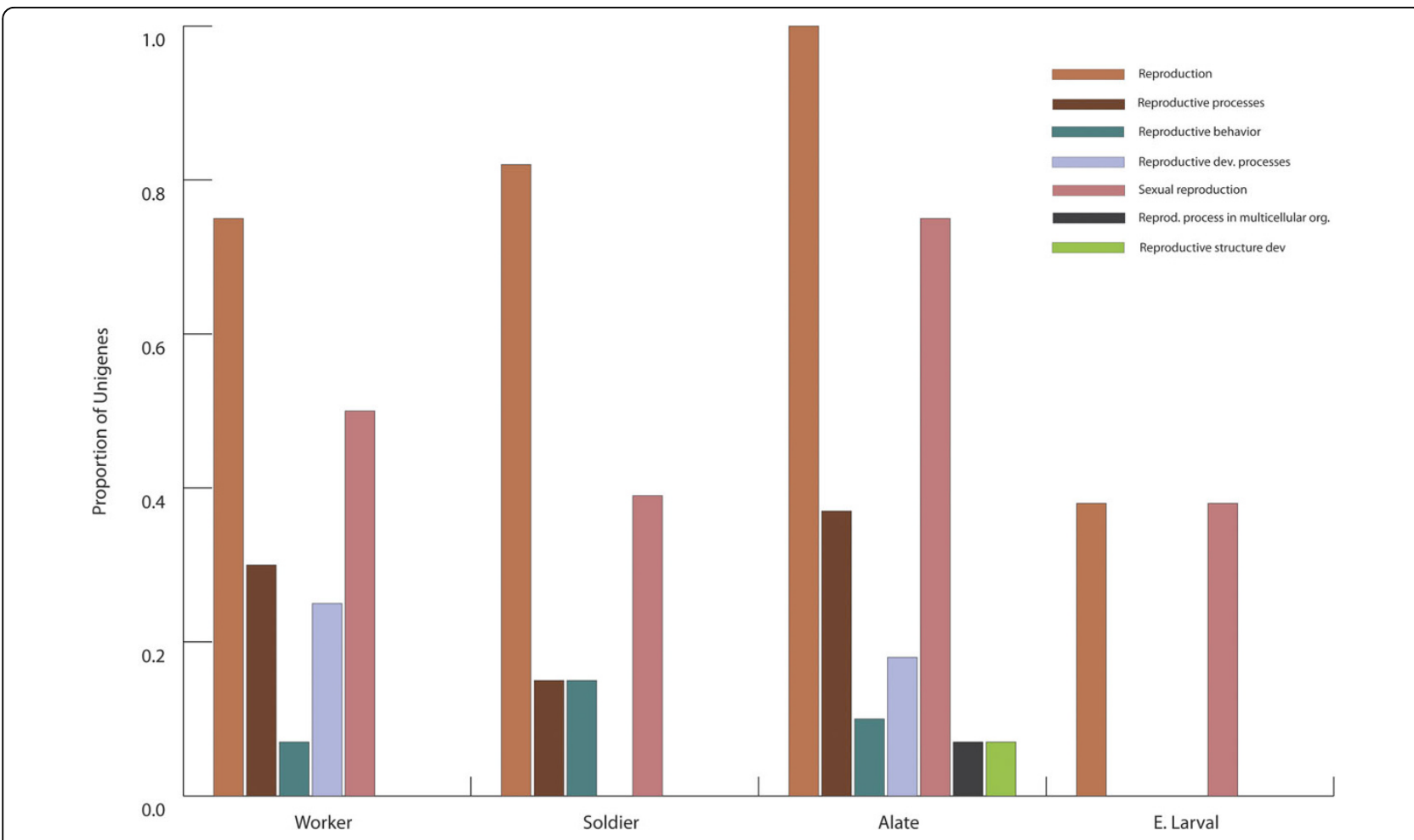

Figure 4 Frequency of genes putatively matching reproductive GO terms in each $R$. flavipes EST library as a fraction to total number of unigenes. No genes with putative reproductive functions were detected in the late larval cDNA library.

reported the presence of this protein in fly heads [21], the female reproductive tract [22], and in response to viral challenge [23]. The pebIII matches a chemosensory protein in A. mellifera (AmelCSP3); however, the expression pattern of this protein in a Northern blot analysis indicated a function in cuticle maturation [24]. The termite sequence was $55 \%$ identical to that of
D. melanogaster (REFSEQ:NP_524966.1; e-value: $2.00 \mathrm{e}^{-28}$ ) and $52 \%$ identical to the $A$. mellifera sequence (e-value: $\left.2.00 \mathrm{e}^{-26}\right)$. It is unknown what role these proteins play in termites, but they are about $97 \%$ similar to each other, with a larger proportion of the transcripts being of alate origin, suggesting the presence of a greater number of transcripts in alates relative to soldiers.

Table 2 In silico analysis of transcript bias in contigs formed from all sequences in each library, the ten highest R-values were analyzed using BLASTX (e-value $\leq 1 \mathrm{E}^{-10}$ )

\begin{tabular}{|c|c|c|c|c|c|c|c|}
\hline Library Bias & Contig \# & Length (bp) & $\mathbf{R}^{1}$ & $p$-value ${ }^{2}$ & Identity & e-value & Identity (\%) \\
\hline Soldier & 3 & 2647 & 106 & $5.24^{\mathrm{e}-50}$ & Viral Protein & $1.00^{\mathrm{e}-170}$ & 44 \\
\hline Soldier & 794 & 1435 & 42 & $4.51^{\mathrm{e}-25}$ & Ejaculatory Bulb-specific Protein 3 & $1.00^{\mathrm{e}-25}$ & 67 \\
\hline Alate & 827 & 1036 & 31 & $1.42^{\mathrm{e}-10}$ & Ejaculatory Bulb-specific Protein 3 & $1.00^{\mathrm{e}-29}$ & 73 \\
\hline Alate & 148 & 981 & 66 & $6.63^{\mathrm{e}-21}$ & ATP Synthase Subunit A & $1.00^{\mathrm{e}-27}$ & 68 \\
\hline Alate & 168 & 1724 & 26 & $1.03^{\mathrm{e}-10}$ & Cyctochrome C Oxidase Subunit 1 & $1.00^{\mathrm{e}-180}$ & 86 \\
\hline Alate & 352 & 1550 & 22 & $1.58^{\mathrm{e}-11}$ & Endoglucanase B Precursor (Cellulase B) & $1.00^{\mathrm{e}-109}$ & 59 \\
\hline Early Larval & 472 & 954 & 92 & $3.61^{\mathrm{e}-48}$ & Cytochrome Oxidase Subunit 3 & $1.00^{\mathrm{e}-86}$ & 76 \\
\hline Early Larval & 479 & 1740 & 74 & $2.82^{\mathrm{e}-35}$ & Cytochrome B & $1.00^{\mathrm{e}-134}$ & 77 \\
\hline Early Larval & 507 & 735 & 40 & $3.24^{\mathrm{e}-20}$ & Tropomyosin & $1.00^{\mathrm{e}-18}$ & 89 \\
\hline Early Larval & 821 & 1349 & 18 & $7.46^{\mathrm{e}-05}$ & Troponin C & $1.00^{\mathrm{e}-38}$ & 70 \\
\hline Late Larval & 412 & 975 & 80 & $3.10^{\mathrm{e}-22}$ & Ribosomal Protein L31 & $1.00^{\mathrm{e}-51}$ & 83 \\
\hline
\end{tabular}

${ }^{1}$ R-statistic was based on equations in Stekel et al. (2000) and compared relative abundance of each libraries prevalence in contiguous sequences from all available sequences.

${ }^{2} p$-values were calculated to establish appropriate significance thresholds to confirm individual library abundance. $p$-value is in reference to significance of caste bias of singletons in contigs formed using a chi-squared test on singleton representation in each contig. 


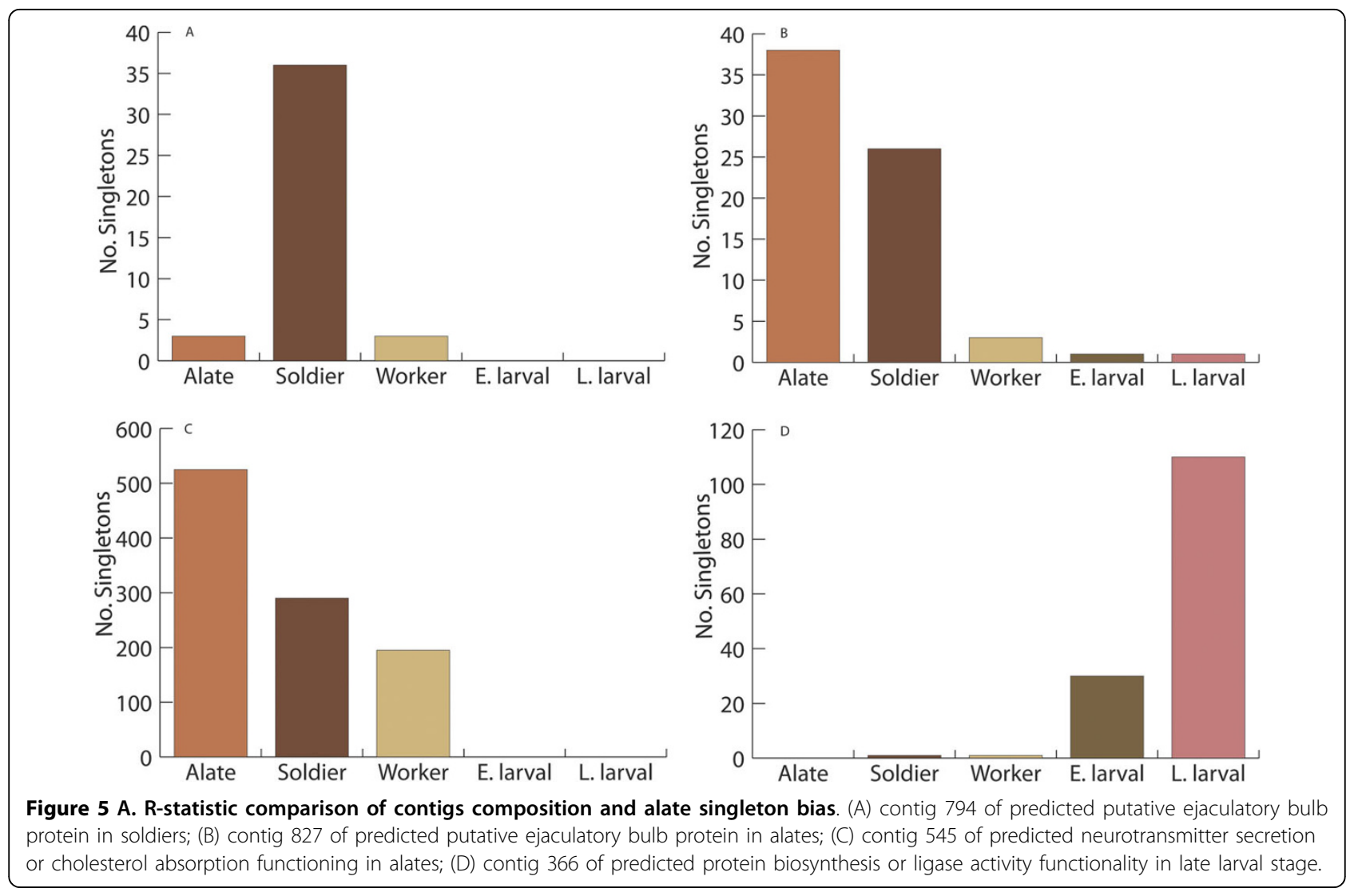

Three other genes showed a significant transcript bias in the alate library. The first gene was most similar to the mitochondrial cytochrome $\mathrm{c}$ oxidase subunit. Higher levels of cytochrome c oxidase expression were previously discovered in dealate ants and hypothesized to be involved in programmed wing cell death [25]. The second gene matched the cellulase $\mathrm{b}$ gene in the bacterium, Cellulomonas firmi; the prevalence of this in alates may reflect higher levels of symbiont load in alates relative to other castes as the gene is of bacterial origin. Alates need extra stores of symbionts to establish the bacteria in the guts of workers and soldiers in a nascent

Table 3 In silico analysis and predicted protein function of 10 contigs with the highest R-value with no putative role assigned by a BLASTX search

\begin{tabular}{cccccc}
\hline Library Bias & Contig \# & Length $(\mathbf{b p})$ & $\mathbf{R}^{\mathbf{1}}$ & $\boldsymbol{p}_{\text {-value }}^{\mathbf{2}}$ & Predicted Protein Function $^{\mathbf{3}}$ \\
\hline Soldier & 910 & 1255 & 15 & $6.92^{\mathrm{e}-11}$ & Antibacterial humoral response; Translation elongation \\
\hline Alate & 545 & 1346 & 382 & $6.39^{\mathrm{e}^{-128}}$ & Neurotransmitter secretion; Cholesterol absorption \\
\hline Alate & 142 & 1745 & 76 & $1.54^{\mathrm{e}-25}$ & Cell adhesion; Hydrolase activity \\
\hline Late Larval & 366 & 478 & 97 & $5.45^{\mathrm{e}-70}$ & Protein biosynthesis; Ligase activity \\
\hline Late Larval & 598 & 903 & 49 & $2.00^{\mathrm{e}-19}$ & Regulation of transcription; Metabolism \\
\hline Late Larval & 516 & 393 & 39 & $2.04^{\mathrm{e}-16}$ & Stress Response \\
\hline Late Larval & 533 & 474 & 31 & $7.12^{\mathrm{e}-13}$ & Cell adhesion \\
\hline Late Larval & 582 & 1133 & 26 & $9.43^{\mathrm{e}-09}$ & Spermatogenesis; Cell-cell adhesion \\
\hline Late Larval & 560 & 197 & 24 & $3.89^{\mathrm{e}-09}$ & Ribosomal protein-nucleus import \\
\hline Late Larval & 596 & 747 & 14 & $1.27^{\mathrm{e}-07}$ & Amino acid metabolism
\end{tabular}

${ }^{1}$ R-statistic was based on equations in Stekel et al. (2000) and compared relative abundance of each libraries prevalence in contiguous sequences from all available sequences.

${ }^{2} p$-values were calculated to establish appropriate significance thresholds to confirm individual library abundance. $p$-value is in reference to significance of caste bias of singletons in contigs formed using a chi-squared test on singleton representation in each contig.

${ }^{3}$ Putative protein functions were predicted using: http://dragon.bio.purdue.edu/pfp. 
colony to assist in cellulose degradation [3]. The final gene matched an ATP synthase subunit suggestive of higher initial energetic needs of colony founders.

The remaining genes analyzed showed biases primarily in the early larval stage library. Most of the genes that matched are involved with general energy production and muscle contraction [26]. It is not clear whether these higher need for energetic reserves and muscle contraction represent the general colony tasks that the larvae perform relative to the more specialized tasks typically undertaken by the older workers and soldiers.

The second group of genes subjected to the R-statistic analysis was of those that showed a library bias of singleton composition but did not return a significant BLAST hit; genes in this group were nonetheless submitted for a putative function prediction based on the protein translation. Our results suggested that soldiers have a higher level of expression of two genes putatively involved in immune function, whereas in other eusocial insects, the pattern of increased immune investment was generally seen in the reproductive individuals [27]. It is important to consider that this sequence was on the lower end of the R-statistic acceptance levels, and thereby its representation was not as biased for one caste or the other relative to those with higher R-statistic values.

The two transcripts with an over-representation in the alate library included proteins putatively involved in neurotransmitter secretion or cholesterol absorption (contig 545), cell adhesion, and hydrolase activity. The over-expression of these genes may indicate a higher level of investment by alates in brain and cell-to-cell communication which may be helpful in mate finding, mate recognition, and colony establishment. Many of the transcripts from late larval stage library have a function putatively associated with energetic needs and metabolism.

Finally, previous studies have implicated two hexamerins $(H e x-I$ and $H e x-I I)$ in soldier-worker differentiation in $R$. flavipes $[28,29]$. We recovered both hexamerin I and II in worker, alate, and early larval stage libraries and hexamerin-I in the soldier library; however they did not exhibit differential expression in the in silico analyses, most likely because we did not examine the transitional form between soldiers and workers, as was done in the previous studies $[28,29]$.

\section{Conclusions}

We presented an analysis of 15,351 randomly chosen cDNA fragments from 5 different cDNA libraries and identified approximately 5,000 ESTs. Relative to the honey bees, little is known about the gene expression differences among various castes and life stages. Therefore, the compiled EST data base will be a valuable resource for future studies on the sociogenomics of termites in general and $R$. flavipes in particular. In addition, the in silico analysis revealed several patterns that could be the subject of future functional genomics studies. For example, the caste-biased transcript abundance of several genes indicates that these proteins may be playing specialized roles in each of the castes or developmental stages. The presence of proteins putatively involved in reproduction in sterile castes is intriguing. It is possible that these proteins play multiple roles or they may be subject to post-transcriptional or posttranslational modifications.

\section{Abbreviations}

cDNA: complementary deoxyribonucleic acid; GO: Gene ontology; rRNA: ribosomal ribonucleic acid.

\section{Acknowledgements}

We thank Michael Scharf for providing termites, advise, and discussion, Joshua Urban for help in the laboratory, and anonymous reviewers for suggestions and constructive criticism. This study was supported by grants from the Ecological Genomics Institute, the Arthropod Genomics Center, and the Center for the Understanding of Origins (all Kansas State University) to SK. This is contribution number 10-082-J of the Kansas Agricultural Experiment Station.

\section{Author details}

${ }^{1}$ Department of Entomology, Kansas State University, Manhattan, KS 66506 USA. ${ }^{2}$ Department of Computing and Information Sciences, Kansas State University, Manhattan, KS 66506, USA.

\section{Authors' contributions}

SK conceived the project; SK and MS undertook laboratory work and data analyses; DC helped with data analysis; SK wrote the paper with help from MS. All authors read and approved the final manuscript.

Received: 28 September 2009 Accepted: 6 August 2010 Published: 6 August 2010

\section{References}

1. Robinson GE, Grozinger CM, Whitfield CW: Sociogenomics: Social life in molecular terms. Nat Rev Genet 2005, 6:257-270.

2. Vargo EL, Husseneder C: Biology of subterranean termites: Insights from molecular studies of Reticulitermes and Coptotermes. Annu Rev Entomol 2009, 54:379-403.

3. Abe T, Higashi T, Bignell D, (eds): Termites: Evolution, Sociality, Symbioses, Ecology. Netherlands: Kluwer Academic Publishers 2000.

4. Austin JW, Szalanski AL, Scheffrahn RH, Messenger MT: Genetic variation of Reticulitermes flavipes (Isoptera: Rhinotermitidae) in North America applying the mitochondrial rRNA 16 S gene. Genetics 2005, 98:980-988.

5. Thorne BL, Traniello JFA, Adams ES, Bulmer M: Reproductive dynamics and colony structure of subterranean termites of the genus Reticulitermes (Isoptera Rhinotermitidae): a review of the evidence from behavioral, ecological, and genetic studies. Ethol Ecol Evol 1999, 11:149-169.

6. Scharf ME, Wu-Scharf D, Pittendrigh BR, Bennett GW: Caste- and development-associated gene expression in a lower termite. Genome Biol 2003, 4:R62

7. Liénard MA, Lassance JXS, Paulmier I, Picimbon J, Lofstedt C: Differential expression of cytochrome c oxidase subunit III gene in castes of the termite Reticulitermes santonesis. J Insect Physiol 2006, 52:551-557.

8. Cornett R, Koshikawa S, Hojo M, Matsumoto T, Miura T: Caste-specific cytochrome P450 in the damp-wood termite Hodotermopsis sjostedti (Isoptera, Termopsidae). Insect Mol Biol 2006, 15:235-244.

9. Hojo M, Koshikawa S, Cornette R, Matsumoto T, Miura T: Identification of soldier-specific genes in the nasute termite, Nasutitermes takasagoensis (Isoptera: Termitidae). Entomol Sci 2005, 8:379-387. 
10. Miura T, Kamikouchi A, Sawata M, Takeuchi H, Natori S, Kubo T, Matsumoto T: Soldier caste-specific gene expression in the mandibular glands of Hodotermopsis japonica (Isoptera: Termopsidae). Proc Natl Acad Sci USA 1999, 96:13874-13879.

11. Sinha S, Ling X, Whitfield CW, Zhai C, Robinson GE: Genome scan for cisregulatory DNA motifs associated with social behavior in honeybees. Proc Natl Acad Sci USA 2006, 103:16352-16357.

12. Evans JD, Wheeler DE: Expression profiles during honeybee caste determination. Genome Biol 2000, 2:research0001.1-0001.6.

13. Cristino AS, Nunes FMF, Lobo CH, Bitondi MMG, Simões ZLP, Da Fontoura Costa L, Evans JD, Hartfelder K: Caste development and reproduction: a genome-wide analysis of hallmarks of insect eusociality. Insect Mol Biol 2006, 15:703-714.

14. Human Genome Center: EGassembler.[http://egassembler.hgc.jp/]

15. Eisenberg E, Levanon EY: Human housekeeping genes are compact. Trends Genet 2003, 19:362-365.

16. Götz S, García-Gómez JM, Terol J, Williams TD, Nagaraj SH, Neuda MJ, Robles M, Talón M, Dopazo J, Conesa A: High-throughput functional annotation and data mining with BLAST2GO suite. Nucleic Acids Res 2008, 36:3420-3435.

17. Stekel DJ, Git Y, Falciani F: The comparison of gene expression from multiple cDNA libraries. Genome Res 2000, 10:2055-2061

18. Kihara Lab of Bioinformatics, Purdue University: Protein Function Prediction.[http://dragon.bio.purdue.edu/pfp/].

19. Scharf ME, Wu-Scharf D, Zhou X, Pittendrigh BR, Bennett GW: Gene expression profiles among immature and adult reproductive castes of the termite Reticulitermes flavipes. Insect Mol Biol 2005, 14:31-44.

20. Bohbot J, Sobrio F, Lucas P, Nagnana-Le Meillour P: Functional characterization of a new class of odorant-binding proteins in the moth Mamestra brassicae. Biochem Biophys Res Comm 1998, 253:489-494.

21. Claridge-Chang A, Wijnen H, Naef F, Boothroyd C, Rajewsky N, Young MW: Circadian regulation of gene expression systems in the Drosophila head. Neuron 2001, 32:657-671.

22. Swanson WJ, Wong A, Wolfner MF, Aquadro CF: Evolutionary expressed sequence tag analysis of Drosophila female reproductive tracts identifies genes subjected to positive selection. Genetics 2004, 168:1457-1465.

23. Sabatier L, Jouanguy E, Dostert C, Zachary D, Dimarcq J, Bulet P, Imler J: Pherokine-2 and -3 two Drosophila molecules related to pheromone/ odor-binding proteins induced by viral and bacterial infections. Eur J Biochem 2003, 270:3398-3407.

24. Forêt S, Wanner KW, Maleszka R: Chemosensory proteins in the honey bee: Insights from the annotated genome, comparative analyses and expressional profiling. Insect Biochem Mol Biol 2007, 37:19-28

25. Tian H, Vinson SB, Coates CJ: Differential gene expression between alate and dealate queens in the red imported fire ant, Solenopsis invicta Buren (Hymenoptera: Formicidae). Insect Biochem Mol Biol 2004, 34:937-949.

26. Parmacek MS, Leiden JM: Structure, function, and regulation of troponin C. Circulation 1991, 84:991-1003.

27. Amdam GV, Simões ZLP, Hagen A, Norberg K, Schroder K, Kirkwood TBL, Omholt SW: Hormonal control of the yolk precursor vitellogenin regulates immune function and longevity in honeybees. Exp Gerontol 2004, 39:767-773.

28. Zhou X, Tarver MR, Bennett GW, Oi FM, Scharf ME: Two hexamerin genes from the termite Reticulitermes flavipes: Sequence, expression, and proposed functions in caste regulation. Gene 2006, 276:47-58.

29. Zhou X, Oi FM, Scharf ME: Social exploitation of hexamerin: RNAi reveals a major caste-regulatory factor in termites. Proc Natl Acad Sci USA 2006, 103:4499-4504.

doi:10.1186/1471-2164-11-463

Cite this article as: Steller et al.: Comparative analysis of expressed sequence tags from three castes and two life stages of the termite Reticulitermes flavipes. BMC Genomics 2010 11:463.

\section{Submit your next manuscript to BioMed Central and take full advantage of:}

- Convenient online submission

- Thorough peer review

- No space constraints or color figure charges

- Immediate publication on acceptance

- Inclusion in PubMed, CAS, Scopus and Google Scholar

- Research which is freely available for redistribution

Submit your manuscript at www.biomedcentral.com/submit 